\title{
Accounting for what counts
}

\author{
Virman Man
}

Published online: 23 October 2010

(C) Springer Science+Business Media B.V. 2010

In the first article in this edition of International Review of Education the action research project presented by Isabel Bartau Rojas and Mariangeles de la Caba Collado examines social inclusion in the context of social disadvantage and dysfunctional families. Using a small sample, the authors address the attitudes and behaviour of parents and children in these families, and in so doing seek to reduce children's learning and behavioural problems. While such change is likely to be a slow and long-term process, readers may reflect that the repetition and extension of the project to a larger population over a longer time span would be likely to throw even more light on the authors' findings, on the change in child-parent dynamics as well as educational outcomes.

Sheryl Feinstein and Lucas Mwahombela report on corporal punishment in Tanzanian schools. They find that despite its outlawing, many well-meaning and dedicated teachers believe that it is necessary. The belief persists that removing corporal punishment means removing all discipline from the classroom. The problem stems partly from Tanzania's drive to enrol more students: class sizes rise to the extent that teachers may be responsible for between 50 and 90 pupils. There is a pressing need to recruit more teachers, who have to be trained to practise alternative strategies for classroom management. In addition, greater public awareness and education about corporal punishment has to be improved, and enforcement of the legislation has to be tightened-up.

Thomas William Nielsen sets out to evaluate the successes and difficulties of a project which uses imagination and emotions as pedagogical tools in making learning more attractive and meaningful to First Nations communities in Canada. The author finds that working with imaginative education is inherently problematic because the affective domain has not been nurtured or encouraged in education. This, combined with LUCID's additional 'ingredient' of cultural inclusion, means

V. Man $(\bowtie)$

UNESCO Institute for Lifelong Learning, Hamburg, Germany

e-mail:v.man@unesco.org 
that assessing links between imaginative teaching, a culturally inclusive curriculum and student learning outcomes presents something of a challenge.

Jianping Shen, Van Cooley, Patricia Reeves, Walter Burt, Lisa Ryan, J. Mark Rainey and Wenhui Yuan deal with leadership and processes for informed decisionmaking - an important topic not only in the Michigan schools that the authors cover, but also, of course, in any organisation. The authors found that it was predominantly the case that student achievement data were used for decision-making by school principals. Other types of data, such as information on the processes schools used to enhance achievement and on student and community background, were more peripheral. The authors argue that by looking at various data streams in conjunction with each other, there could be marked improvements in decision-making.

In a very welcome contribution, Ahmad Oweini and Katia Hazoury tackle a rarely touched-upon subject. International test comparisons show that students in the Arab world have difficulties in learning to read and have limited comprehension to advanced grades. By developing a sight-list of the most frequently used Arabic word forms, the authors propose that it is more likely to be able to achieve reading automaticity and fluency at an early stage. Those who are familiar with the orthography of the Arabic language might suggest that it particularly lends itself to such a mechanical treatment of reading. Furthermore, it would be interesting to conduct some research to see what cross-cultural comparisons might reveal. It is to be hoped that publication of the article will help to stimulate further contributions to this journal from this under-represented region.

It would be a truism to say that authors who have had more publishing success tend to be those who have had most access to journals, for reasons of cost, availability or language. Thus, scholars in Europe and North America, for example, are more likely to be published — not because they are better academics, but simply because they are more familiar with the rules of the game. Moreover, with the increasing preference of journal publishers to use English as the medium of publication, this scenario is set to continue. The clear danger is that what counts as valid knowledge is limited to that which can navigate its way through the publishing process: a process which favours one part of the world over another.

Questions of access to publishing in academic journals were addressed at two conferences this year, the 54th Annual Conference of the Comparative and International Education Society (Chicago, March) and the 14th Congress of the World Council of Comparative Education Societies (Istanbul, June). Both events featured sessions dedicated to "meeting the editors", at which authors (whether published or unpublished) were invited to raise questions about the publishing process. Interesting and varied subjects were aired, including editorial policy, peer review procedures, the relative advantages and disadvantages of online and print publication, and how the language of publication affects not only readership but also the likelihood of being published. These sessions were an opportunity for a valuable exchange of views from both sides of the fence, as it were.

Individual academics and their institutions are under increasing pressure to publish, as volume of published output is often taken to be a measure of research quality. This in turn exerts a squeeze on journals, which have a limited number of editions and pages. The task for editors, who are effectively gatekeepers of what 
gets published, is to select from the huge quantity of articles received those which they believe to be of interest to their journal's readership. Inevitably, many submissions are rejected, for varying reasons: the subject matter may fall outside the journal's editorial remit; the article contributes nothing new or goes over old ground; it may not be internally consistent; the evidence to support conclusions is insufficient; or simply it is poorly written. Sometimes publication takes place only after an iterative process in which authors are asked to revise and re-submit. It can be frustrating and time-consuming.

It was absorbing to hear how other editors and journals try to grapple with these tensions, and it would be fair to say that none could point to a straightforward solution. For its part, the International Review of Education's Editorial Board, meeting in April this year, strongly endorsed continuing efforts to encourage submission from countries in the south, to ensure that other voices and experiences are heard. Papers by authors based in or from the south do not have an automatic wave-through to publication, however, and like all submissions still have to satisfy peer-reviewers on standard quality criteria.

As the content of this edition illustrates, something else which the International Review of Education has in common with many of the journals represented at the conferences mentioned earlier is its eclecticism. Its breadth of coverage encompasses policy, practice and research in education and learning at all stages of life, in all areas of the world. It publishes work at all scales of analysis, from a local community to single-country studies and international comparisons. It covers not only formal education but also non-formal and informal modes of education and learning. So, what is it that binds such seemingly different topics and approaches together? Arguably, it is the fact that articles report on or analyse how educational policy is enacted in different geographical (or historical) contexts. An essay based on experiences in one region or country can have as much validity as a crosscountry analysis. Both can contribute to knowledge and understanding of how policy is translated into practical action, and the effects of that action. If there are new perspectives or fresh information, or if techniques and approaches can be applied in different settings, then they are indeed useful additions to the literature.

As a final point of information, readers might like to note that Hannah Mowat has left the Editorial Office and that a new Assistant Editor will be in post before the next edition of IRE. 